\title{
Routine Screening for HIV Infection in a Low Risk Population
}

\author{
Mary Bessesen $^{1,2}$, Patricia Stamper ${ }^{1}$, James Shaw ${ }^{1}$, Neelam Ojha ${ }^{1}$, Anthony Currans ${ }^{1}$, \\ Don Weinshenker ${ }^{1,2}$, Daniel Merrick ${ }^{1,3}$ \\ ${ }^{1}$ Department of Veterans Affairs, Eastern Colorado Healthcare System, Denver, USA; ${ }^{2}$ Department of Medicine, School of Medicine, \\ University of Colorado-Denver, Denver, USA; ${ }^{3}$ Department of Pathology, School of Medicine, University of Colorado-Denver, Den- \\ ver, USA. \\ Email: Mary.Bessesen@ucdenver.edu
}

Received January $12^{\text {th }}, 2012$; revised February $13^{\text {th }}, 2012$; accepted March $15^{\text {th }}, 2012$

\begin{abstract}
Context: Despite advances in therapy for HIV infection, treatment is often compromised by delayed diagnosis. Strategies are needed to improve broad based testing in all medical settings. Objective: To compare HIV screening rates in a primary care setting following implementation of an electronic reminder system to rates produced solely by provider educational efforts. Design: Prospective, observational study of HIV screening, comparing rates during implementation of strategies. Setting: Multiple clinic healthcare system affiliated with a tertiary care medical center. Patients: Veterans receiving care in the clinics affiliated with the Department of Veterans Affairs-Eastern Colorado Healthcare System. Intervention: Provider education and a provider clinical reminder system embedded in the electronic medical record. Main Outcome Measure: Proportion of primary care clinic appointments at which the veteran was screened for HIV infection. Results: The proportion of visits at which screening occurred increased from $2.7 \%$ during the provider education period to $16.7 \%$ during the provider clinical reminder period $(\mathrm{p}<0.0001)$. All of the cases identified by the reminder were antiretroviral therapy candidates, and there was a trend to earlier diagnosis in the screening group than in the diagnostic testing group (CD4 count 329 vs. $109, p=0.13$ ). Conclusions: An electronic clinical reminder system in a primary care setting is an effective strategy to increase testing for HIV infection, and may lead to fewer delayed diagnoses.
\end{abstract}

Keywords: HIV; HIV Infection; Screening

\section{Introduction}

There has been tremendous progress in the management of HIV infection since 1981, when the acquired immunodeficiency syndrome was first reported. In the absence of therapy, HIV infection progresses to AIDS in approximately 10 years, but currently a 25 year-old person with newly diagnosed HIV infection, and access to high quality care, has a life expectancy of 64 years [1]. Despite advances in therapy, and the benefit of early treatment, early diagnosis of HIV infection has been an elusive goal. A recent CDC-funded study of opt-out testing found a median CD4 positive T lymphocyte count of $69 / \mathrm{uL}$ among the newly diagnosed cases identified by routine screening [2].

Early diagnosis and treatment of HIV infection is also an important part of the national HIV prevention strategy [3]. Persons who are aware of their infection are less likely to transmit infection to others [4], because they can adopt less risky behaviors, and antiretroviral therapy of
HIV-infected persons is an effective prevention strategy. Treatment with antiretroviral agents reduces viral burden in blood and in genital secretions, and in serodiscordant heterosexual couples highly active antiretroviral treatment of the infected partner reduces transmission of HIV $96 \%$ [5].

It is estimated that 1.2 million Americans are infected with human immunodeficiency virus (HIV), and that $20 \%$ of infected persons are unaware of their infection [6]. In 2006, the Centers for Disease Control published updated guidelines for HIV testing, that recommended all persons younger than 65 years be tested at least once, regardless of risk factors [7], and that screening be incorporated into routine medical care, without a requirement for formal pre-test counseling or written informed consent. Despite these guidelines, the rate of new diagnoses of HIV infection reported to CDC did not increase from 2006 through 2009 [6]. Furthermore, the AIDS mortality rate in the United States did not change be- 
tween 2006 and 2008, and late diagnosis of HIV infection has improved only modestly [3].

Previous efforts to increase HIV testing have focused on relatively high risk populations, e.g. patients presenting to Sexually Transmitted Disease clinics and Emergency Departments in urban safety net hospitals $[2,8,9]$. To achieve the goal of testing all Americans between 13 and 65 years old, screening must be included in routine medical care for populations that are not perceived to be at increased risk of HIV infection. To address this need, a program was developed to improve testing within the Veterans Affairs Eastern Colorado Healthcare System.

Legal requirements for written informed consent for HIV testing in the Department of Veterans Affairs were an impediment to the normalization of HIV testing until 2009 , when a Congressional action allowed a change in policy. In August, 2009, the requirement for written informed consent for HIV testing was rescinded, and providers were advised of the recommendation that all patients be tested, following patient education and verbal consent. In March 2010, enhanced educational efforts were directed to providers and patients, to encourage broad based testing of all patients, regardless of risk factors. In June, 2010, a clinical reminder for HIV testing was designed and embedded in the electronic medical record at the Department of Veterans Affairs Eastern Colorado Healthcare System. We analyzed testing rates and results associated with each of these efforts.

\section{Methods}

\subsection{Setting}

The Eastern Colorado Healthcare System provides primary and specialty care to 70,000 veterans in eight community based outpatient clinics and a tertiary medical center with 92 inpatient beds and a full range of subspecialty clinics, staffed by physician faculty at the University of Colorado-Denver Medical School. The HIV treatment program is staffed by one nurse practitioner and three Infectious Diseases specialists.

\subsection{Testing Programs}

Baseline testing program: Until August, 2009, HIV testing was available when a physician or nurse provided pre-test counseling, ordered the test, and a written informed consent form signed by the patient was delivered to the laboratory with the blood specimen.

Verbal consent program: In August, 2009, a local policy was approved, which encouraged HIV testing, and required only that the ordering physician or nurse obtain verbal consent and provide written educational materials to the patient before ordering an HIV screening test. The new policy was circulated to providers by electronic mail.

Educational program: Educational posters and pamphlets were placed at all clinic check-in areas. The medical director of the Infectious Diseases clinic attended staff meetings for primary care providers, and provided information on the $\mathrm{CDC}$ recommendations for testing, and the amended consent rules. The Infectious Diseases Nurse Practitioner visited nursing meetings to provide the same information. In addition, she attended general medical intake clinics for new patients to the system bimonthly, providing care to new patients, and setting an example of HIV testing of all patients. The educational message on the telephone "on hold" system was amended to include the statement, "Did you know that the Centers for Disease Control recommend that all adults be tested at least once for HIV infection? Everyone, not just people who are at high risk, should be tested at least once. For people who engage in high risk behaviors, more frequent HIV testing is recommended. Even if you do not think you are at risk you should talk to your provider about taking the HIV test."

Clinical reminder program: All patient records are maintained in a comprehensive electronic medical record, which has been in use since 1998. Providers enter progress notes into the electronic medical record at each patient encounter. A clinical reminder system is embedded in the progress note program, and is used to alert providers to a variety of health maintenance tasks, e.g. colorectal cancer screening for persons over 50 years of age. We designed and implemented a clinical reminder that searched the medical record for a previous HIV antibody test result, and alerted the provider if no result was found. The provider would then offer HIV testing to the patient, and with two mouse clicks the order was entered and documented in the record. The reminder was activated for patients of all ages. Providers could complete the reminder by entering results of testing performed outside of the VA, or by indicating that life expectancy was less than six months. All of the educational efforts described above continued during the clinical reminder period.

A screening ELISA was performed, using the Abbott HIVAB HIV-1/HIV-2 (rDNA) EIA kit. Samples that tested positive were sent to a reference laboratory (LabCorp), for confirmatory testing. The ELISA was repeated at the reference laboratory, and if it was positive, a Western Blot was performed (Figure 1). Each case with a positive screening ELISA was reviewed, and classified as a true positive or false positive, based on the result of the confirmatory ELISA and Western Blot assays. All patients who had a positive or indeterminate Western Blot assay also had a quantitative HIV PCR performed, using the COBAS Amplicor assay (10/1/2007-7/31/2009), or the Roche Amplicor/COBAS Taqman HIV-1 assay (8/1/2009 
-11/30/2010). The laboratory alerted the Infectious Diseases team to each positive screening ELISA test, at the time that the specimen was sent to the reference laboratory for confirmatory testing. The Infectious Diseases team (MTB and PS) performed a chart review of each case with a positive screening ELISA, and informed the primary care provider of the screening test result. If the screening test was confirmed by a positive Western Blot assay, the team provided assistance to the primary care provider with counseling the patient about the test result. Each newly diagnosed case was followed prospectively, clinical features were recorded on a standardized case report form, and the case was classified as below.

1) Screening test-a test ordered by a provider in a case in which no risk factors or clinical signs or symptoms of HIV infection were recorded in the provider's note or on the problem list.

2) Reminder-prompted test-a screening test, performed when the provider processed the clinical reminder.

3) Diagnostic test-a test performed in a case in which the provider suspected HIV infection, based on patient report of a risk factor or clinical signs and symptoms.

4) Confirmatory test-a test ordered to confirm a history of HIV infection.

\subsection{Analysis}

We analyzed screening rates during four periods, Period 1, Baseline period, 10/1/2007-8/31/2009; Period 2, Verbal consent period, 9/1/2009-2/28/2010; Period 3, Educational program period, 3/1/2010-5/31/2010; Period 4, Clinical Reminder Period, 7/1/2010-11/30/2010. We retrieved the number of clinic visits for each period from administrative records. The clinical reminder was activated on $6 / 20 / 2010$. We therefore excluded the month of June from analysis of testing rates, because it included tests performed under both educational program and clinical reminder program conditions.

Statistical analyses were performed on GraphPad InStat, using chi square or Fisher's exact test for comparisons of proportions, and Student t-test for continuous variables.

\section{Results}

There were 8528 initial screening ELISA assays performed between March 1, 2010 and November 30, 2010, encompassing the educational and clinical reminder periods (Figure 1). There were five positive tests that were ordered to confirm a previously reported diagnosis of HIV infection, and four positive tests performed for diagnostic reasons. These were excluded from the analysis of sensitivity, specificity and predictive value, because they were not ordered for screening purposes. There were 18 positive screening ELISA tests, of which 6 were confirmed positive by repeat ELISA and Western Blot testing, 11 were negative on repeat ELISA testing, and one had a positive repeat ELISA, and an indeterminate Western Blot. Follow-up of the case with an indeterminate Western Blot with an HIV PCR and T-lymphocyte subset analysis demonstrated no evidence of HIV infection. We therefore had 12 false positive screening ELISA tests among 8519 tests run, for a specificity of $99.86 \%$. The predictive value of a positive screening ELISA in this low risk population was $6 / 18=33 \%$. The predictive value of a positive two-step ELISA was $6 / 7=86 \%$.

There were 7220 HIV tests performed during the clinical reminder period, a six fold increase in the monthly test rate when compared to the educational program period, and a ten fold increase compared to the baseline period (Table 1). The proportion of primary care clinic visits at which an HIV test was performed increased from

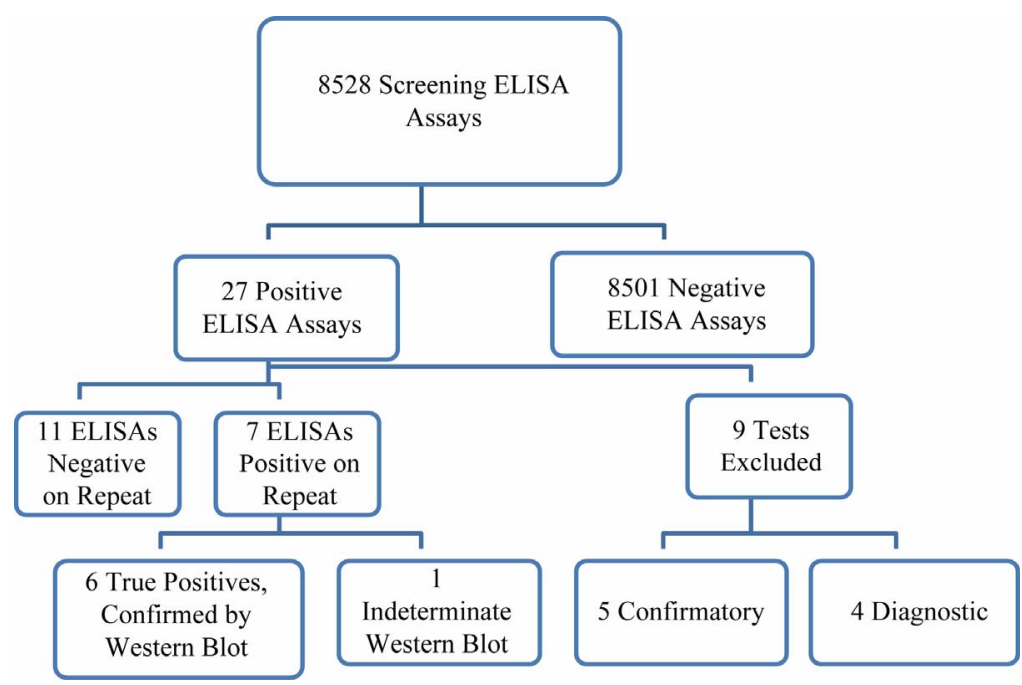

Figure 1. Flow chart of HIV Testing Cascade. 
Table 1. Testing rates.

\begin{tabular}{lcc}
\hline \multicolumn{1}{c}{ Period } & $\begin{array}{c}\text { HIV Tests per } \\
\text { month }\end{array}$ & $\begin{array}{c}\text { HIV Tests per } \\
\text { Clinic Visits }\end{array}$ \\
\hline 1) Baseline & 83 & $1.5 \%$ \\
2) Verbal consent & 141 & $2.0 \%$ \\
3) Educational Program & 233 & $2.7 \%$ \\
4) Clinical Reminder & 1444 & $16.7 \%$ \\
\hline
\end{tabular}

$2.7 \%$ during the baseline period to $16.7 \%$ during the clinical reminder period $(\mathrm{p}<0.0001)$.

Among the 15 patients with a positive Western Blot assay, confirmatory testing with HIV PCR was positive in all cases. Five of the positive ELISA tests were performed to confirm a reported history of HIV infection, and four tests were run for diagnostic reasons. The mean CD4/CD8 positive $\mathrm{T}$ lymphocyte count of the cases tested for diagnostic reasons was 109/uL (range 6 - 398); the median plasma HIV RNA was 56,550 (Table 2). Six of the positive ELISA tests were true screening tests, run in response to the clinical reminder. The mean CD4/CD8 positive $\mathrm{T}$ lymphocyte count of the cases identified by screening patients without identified risk factors was 329/uL (range 41 - 496); the median plasma HIV RNA was 26,400 (Table $3, p=0.13$ ). One of six patients iden- tified by screening had an AIDS diagnosis, vs. three of four patients identified by diagnostic testing (Relative risk $=0.3000,95 \%$ Confidence Interval: 0.05291 to $1.701, \mathrm{p}=0.30$ ).

Linkage to care: All patients were referred to the Infectious Diseases clinic; the median time between the HIV test order date and Infectious Diseases clinic visit date was 15.5 days. The median time between HIV Western Blot assay result date and Infectious Diseases clinic visit was 2 days.

Mental Health Referrals: Referral to the Mental Health service for acute counseling was provided to $50 \%$ of the patients detected by screening prompted by the clinical reminder, compared to none of the patients tested diagnostically. One patient required admission to the inpatient psychiatric service for management of acute adjustment disorder and suicidal ideation.

Costs: The cost to the laboratory is $\$ 8.00$ per HIV antibody test. If we ascribe all HIV tests performed during the clinical reminder period to the reminder, the laboratory cost of the reminder strategy was $\$ 57,760$, or $\$ 9627$ per case detected.

\section{Discussion}

This study demonstrates that routine screening of lowrisk patients in an outpatient setting yields a small, but

Table 2. Characteristics of cases detected by diagnostic HIV testing.

\begin{tabular}{|c|c|c|c|c|c|}
\hline Case & CD4 & Viral load & Reason for test & Risks reported before test & Risks reported after test \\
\hline 1 & 6 & 11,500 & Oral lesion (Kaposi Sarcoma) & None & MSM \\
\hline 2 & 23 & 11,400 & Wasting & None & MSM \\
\hline 3 & 398 & 2450 & Vocal cord papilloma & None & None \\
\hline 4 & 10 & 41,300 & Cholestatic jaundice & None & MSM \\
\hline Mean $+/-$ std dev & $109+/-193$ & & & & \\
\hline median & 16.5 & 56,550 & & & \\
\hline
\end{tabular}

Table 3. Characteristics of cases detected by clinical reminder.

\begin{tabular}{|c|c|c|c|c|c|c|c|}
\hline Case & Age & $\mathrm{CD} 4$ & Viral load & Time to follow-up & Time to ARV & $\begin{array}{c}\text { Risks reported } \\
\text { before test }\end{array}$ & $\begin{array}{l}\text { Risks reported } \\
\text { after test }\end{array}$ \\
\hline 1 & 51 & 366 & 11,500 & 15 & 53 & None & MSM \\
\hline 2 & 27 & 369 & 11,400 & 3 & 36 & None & MSM \\
\hline 3 & 46 & 496 & 2450 & 1 & 42 & None & None \\
\hline 4 & 56 & 224 & 41,300 & 0 & 15 & None & MSM \\
\hline 5 & 38 & 41 & 52,300 & 11 & 22 & None & Prostitute contact \\
\hline 6 & 58 & 280 & 77,300 & 0 & 48 & None & MSM \\
\hline Mean $+/-$ std dev & & $329+/-172$ & & & & & \\
\hline median & 48.5 & 323 & 26,400 & 2 & 39 & & \\
\hline
\end{tabular}

MSM: Men who have sex with men. 
clinically important number of HIV cases that were not detected by a risk-based screening strategy. Furthermore, a clinical reminder system resulted in a marked increase in screening, compared to a strategy of provider education. All of the cases detected by screening met current criteria for antiretroviral therapy, but only $17 \%$ had an AIDS diagnosis, compared to $75 \%$ of the cases detected by diagnostic testing. Therefore, routine screening of a low-risk clinic population may help reach the goal of earlier diagnosis of HIV infection.

Linkage to care was excellent in the screened population, with a median time to first visit with the Infectious Diseases service of only two days. This was achieved through a close relationship with the clinical laboratory, with direct reporting of positive results to the Infectious Diseases service. It is notable that a large proportion of the cases detected by screening required intervention from the Mental Health service. There were no suicide attempts in this small group of patients, which is similar to findings of larger studies. Although a retrospective study in New York found an increased risk of suicide in men with a new diagnosis of HIV infection, a national retrospective study, and a prospective study of military recruits showed no increased risk [10-12]. Patients who present with symptoms of infection or who recognize their risk and request testing may be better prepared for a positive result than persons detected by screening. Although verbal pre-test counseling and written educational materials were provided to all patients prior to testing, it was not the primary reason for their visit on the day of testing. In many cases it was one of several screenings provided at the visit. Implementation of broad based screening of low risk populations will require institutional support and commitment to provide Mental Health services as needed to patients who test positive.

A formal cost effectiveness analysis was beyond the scope of this study, but previous authors have found that when routine HIV screening is cost-effective when incidence is greater than $0.05 \%$ [13]. Specificity of the screening protocol was excellent, leading to a good positive predictive value, despite the low pre-test probability. The predictive value of the initial screening ELISA was $33 \%$, and when the repeat ELISA strategy was applied the positive predictive value increased to $86 \%$. To put this in context, fecal occult blood testing has a positive predictive value of $8 \%$ for colorectal cancer, and $32 \%$ for advanced adenoma [14]. The availability of advanced testing for HIV infection, including polymerase chain reaction for the presence of the virus, removes the ambiguity from cases with an indeterminate Western Blot assay, allowing patient reassurance during the follow-up period.

This study builds on previous work by many centers to reduce the number of HIV-infected persons who are unaware of their infection $[2,8]$. In 2005 a clinical reminder was developed at another VA facility, which used data available in the electronic medical record to identify patients at increased risk for infection, and alert providers to request an HIV test [15]. Our study is the first to focus exclusively on a population with no known risk factors, and a low prevalence of HIV infection.

The limitations of this study are that although there was a trend toward earlier diagnosis during the clinical reminder period, the differences in CD4 positive $\mathrm{T}$ cell numbers did not achieve statistical significance. The relatively small number of HIV infections detected probably resulted in inadequate power to detect a difference. We also could not calculate the sensitivity of the screening test strategy in our population, because we did not apply an independent gold standard test for HIV infection, e.g. HIV PCR, to the patients with negative screening tests. Based on published data, we presume that the sensitivity of the screening test approaches $100 \%[16,17]$. The strength of this study is the large number of patients screened, in a routine clinical care setting, with minimal resources. The provider clinical reminder is a sustainable intervention that can be implemented at low cost by practices that use an electronic medical record and can efficiently achieve linkage to medical and mental health care.

\section{Acknowledgements}

The authors report no conflicts of interest. Mary $T$. Bessesen had full access to all of the data in the study and takes responsibility for the integrity of the data and the accuracy of the data analysis. This study was approved by the Colorado Multiple Institutional Review Board.

\section{REFERENCES}

[1] N. Lohse, A. B. Hansen, G. Pedersen, et al., "Survival of Persons with and without HIV Infection in Denmark, 1995-2005," Annals of Internal Medicine, Vol. 146, No. 2, 2007, pp. 87-95.

[2] J. S. Haukoos, E. Hopkins, A. A. Conroy, et al., "Routine Opt-Out Rapid HIV Screening and Detection of HIV Infection in Emergency Department Patients," Journal of the American Medical Association, Vol. 304, No. 3, 2010, pp. 284-292. doi:10.1001/jama.2010.953

[3] Centers for Disease Control and Prevention, "Vital Signs: HIV Testing and Diagnosis among Adults - United States, 2001-2009," Morbidity \& Mortality Weekly Report, Vol. 59, No. 47, 2010, pp. 1550-1555.

[4] G. Marks, N. Crepaz and R. S. Janssen, "Estimating Sexual Transmission of HIV from Persons Aware and Unaware That They Are Infected with the Virus in the 
USA,” AIDS, Vol. 20, No. 10, 1920, pp. 1447-1450.

[5] J. Castilla, J. Del Romero, V. Hernando, B. Marincovich, S. Garcia and C. Rodriguez, "Effectiveness of Highly Active Antiretroviral Therapy in Reducing Heterosexual Transmission of HIV," Journal of Acquired Immune Deficiency Syndromes, Vol. 40, No. 1, 2005, pp. 96-101. doi:10.1097/01.qai.0000157389.78374.45

[6] Centers for Disease Control and Prevention, HIV Surveillance Report 21, 2010. http:// www.cdc.gov/hiv/resources/factsheets/us.htm

[7] B. M. Branson, H. H. Handsfield, M. A. Lampe, et al., "Revised Recommendations for HIV Testing of Adults, Adolescents, and Pregnant Women in Health-Care Settings," Morbidity \& Mortality Weekly Report Recommendations \& Reports, Vol. 55, No. RR-14, 2006, pp. 1-17.

[8] R. C. Merchant, G. R. Seage, K. H. Mayer, M. A. Clark, V. G. DeGruttola and B. M. Becker, "Emergency Department Patient Acceptance of Opt-In, Universal, Rapid HIV Screening," Public Health Reports, Vol. 123, Suppl. 3, 2008, pp. 27-40.

[9] S. R. Kendrick, K. A. Kroc, E. Couture and R. A. "Weinstein. Comparison of Point-of-Care Rapid HIV Testing in Three Clinical Venues," AIDS, Vol. 18, No. 16, 2004, pp. 2208-2210. doi:10.1097/00002030-200411050-00017

[10] P. M. Marzuk, H. Tierney, K. Tardiff, et al., "Increased Risk of Suicide in Persons with AIDS," Journal of the American Medical Association, Vol. 259, No. 9, 1988, pp. 1333-1337. doi:10.1001/jama.1988.03720090023028

[11] T. R. Cote, R. J. Biggar and A. L. Dannenberg, "Risk of Suicide among persons with AIDS," Journal of the American Medical Association, Vol. 268, No. 15, 1992, pp. 2066-2068. doi:10.1001/jama.1992.03490150118035

[12] A. L. Dannenberg, J. G. McNeil, J. F. Brundage and R. Brookmeyer, "Suicide and HIV Infection. Mortality Follow-Up of 4147 HIV-Seropositive Military Service Applicants," Journal of the American Medical Association, Vol. 276, No. 21, 1996, pp. 1743-1746.

[13] G. D. Sanders, A. M. Bayoumi, V. Sundaram, et al., "Cost-Effectiveness of Screening for HIV in the Era of Highly Active Antiretroviral Therapy," New England Journal of Medicine, Vol. 352, No. 6, 2005, pp. 570-585. doi:10.1056/NEJMsa042657

[14] N. Segnan, C. Senore, B. Andreoni, et al., "Randomized Trial of Different Screening Strategies for Colorectal Cancer: Patient Response and Detection Rates," Journal of the National Cancer Institute, Vol. 97, No. 5, 2005, pp. 347-357. doi:10.1093/jnci/dji050

[15] M. B. Goetz, T. Hoang, C. Bowman, et al., "A System-Wide Intervention to Improve HIV Testing in the Veterans Health Administration," Journal of General Internal Medicine, Vol. 23, No. 8, 2008, pp. 1200-1207. doi:10.1007/s11606-008-0637-6

[16] F. Ketema, C. Zeh, D. C. Edelman, R. Saville and N. T. Constantine, "Assessment of the Performance of a Rapid, Lateral Flow Assay for the Detection of Antibodies to HIV," Journal of Acquired Immune Deficiency Syndromes, Vol. 27, No. 1, 2001, pp. 63-70. doi:10.1097/00126334-200105010-00011

[17] Abbott Laboratories, "HIVAB HIV-1/HIV-2 (rDNA) EIA Package Insert," 2011. http://www.fda.gov/downloads/BiologicsBloodVaccines/ BloodBloodPrducts/ApprovedProducts/LicensedProducts BLAs/BloodDonorScreening/InfectiousDisease/UCM091 153.pdf 